\title{
Editorials
}

Kari G. Smedstad MB CHB FRCPC McMaster University, Hamilton, Ontario

\section{Infection after central neuraxial block}

reports, they have little in common. Some patients are previously healthy and young, (but pregnant), others are sick and have multisystem disease. We need to keep the health status of the patient in mind when we use epidural and or spinal anaesthesia, and be alert to the greater possibility of infection in debilitated, immunocompromised or diabetic patients. However, these disease states are not, in themselves, contraindications to the technique; quite the opposite in many cases. Bromage recommended the use of a medic-alert system for anyone who had received epidural steroids ${ }^{4}$ but the economic and practical feasibility of this was disputed in the accompanying editorial. ${ }^{3}$ General vigilance and clinical examination may well suffice. Cases of late epidural abscess with bad outcome seem to be related to a low index of suspicion with delay in diagnosis and treatment. Thus, prevention of bad outcome can be achieved, even if the infection itself cannot be prevented.

The extrinsic factors should be examined carefully. The present case and others state categorically that full aseptic precautions were taken. This included the use of scrub, gloves, gown and mask by the practitioner, and aseptic preparation of the patient using standard surgical antiseptic solutions which were allowed to dry before proceeding with the needle stick. Yet infection still occurred. Is there a relationship between the technique and epidural abscess formation or meningitis? $\mathrm{A}$ recent survey of British obstetrical anaesthetists confirmed that the practice of wearing masks varies. ${ }^{11}$ There is no information about any relationship between the wearing of masks and the incidence of infection.

McMaster University has just completed a randomized trial of surgical scrubbing with a brush compared with the use of antiseptic soap alone. No scrub brushes have been used in our operating rooms for one year for any procedure. The incidence of postoperative infection did not change. Some individuals who washed with soap alone had a greater reduction in bacterial counts of their hands than those who scrubbed. ${ }^{12}$ I tried to find information on randomized studies of the efficacy of bacterial filters on epidural catheters. The filters are useful for eliminating parti- 
cles such as glass, but I was unable to establish whether they prevent bacterial contamination and thus abscess formation in the epidural space. The antiseptic solution should be sterile. Multiuse bottles of iodine can be contaminated and polyurethane dressings over epidural puncture sites may augment sweating and increase bacterial counts. We use micropore tape swabbed with alcohol to cover our epidural sites. It sticks like glue for days, and allows the skin to "breathe." The issue of duration of use for epidural catheters is also cloudy. We leave the catheter in place for post-operative pain relief until the patient can take oral medications and this averages four days. We insert about 10 epidurals a week for pain relief after surgery. In nine years we have not seen an epidural abscess in any patient. Cancer patients sometimes have epidural catheters in place for weeks.

Common sense dictates that one should use a sterile technique when entering the central neural spaces. In our practice, that means using mask and gloves after washing one's hands well with antiseptic soap solution and drying them on a sterile towel. In the OR, one uses greens but not necessarily a sterile gown. The patient's back is prepared with a sterile antiseptic solution which should be left to dry, then wiped off to prevent introducing the chemical into the epidural space. If multiple needle sticks and tissue trauma occur, it may be wise to change the needle before entering the epidural or subarachnoid spaces. I prefer to use a bacterial filter, particularly if the catheter stays in place for days and multiple injections are given through it. We then change the filter every $48 \mathrm{hr}$. There is a need for studies proving the efficacy of these filters in preventing infections.

Before these procedures are carried out, the patients need to be informed of potential risks and benefits. Informed consent requires the disclosure of material risks. Infection with subsequent neurological sequelae is a material risk, albeit very rare. My practice, when the patient asks, is to tell them that there is always a very remote risk of infection or haemorrhage associated with epidural and spinal techniques, but that the benefits far outweigh the risks. In Canada, specific consent is unnecessary for the administration of anaesthesia for surgery. The preoperative visit provides an opportunity for discussion about alternative forms of anaesthesia, and regional techniques are properly discussed here. No cases of epidural abscess associated with anaesthesia or pain relief techniques have resulted in lawsuits in Canada.

The case reports to date alert us to the fact that infection can and does occur with epidural and spinal anaesthesia. We should take all reasonable precautions to prevent infection, be aware of which patients are most at risk, and follow our patients carefully. Susceptible patients should be told the signs of infection when leaving the hospital setting, because many epidural abscesses present late. Appropriate early diagnosis and treatment of this medical emergency is essential to prevent disastrous sequelae.

\section{L'infection après l'anesthésie régionale centrale}

L'abcès épidural et la méningite consécutives à l'anesthésie régionale centrale sont extrêmement rares. Cependant, nos publications en font de plus en plus mention. On ne sait trop si c'est dû au fait que l'incidence de ces complications augmente ou au fait que les intéressés sont plus enclins à les rapporter.

Dans ce numéro, Schröter et al. rapportent un abcès épidural survenu après un bloc combiné spinalépidural (CSE) chez un patient de 72 souffrant d'insuffisance coronaire et vasculaire périphérique. ${ }^{1}$ On réalise maintenant qu'il ne s'agit pas là d'un cas isolé. Ceci ne signifie pas nécessairement que cette technique augmente le risque d'infection mais il faut au moins savoir que l'infection est possible. Ces cinq dernières années, plusieurs éditoriaux nous ont mis en garde contre la possibilité d'une infection après l'anesthésie épidurale et sous-arachnoïdienne. ${ }^{2-4}$

L'abcès épidural survient aussi bien chez des sujets bien portants que chez des sujets débilités. Il a été rapporté après une anesthésie générale au cours de laquelle on n'avait pas ponctionné l'espace épidurale ou sous-arachnoïdien. ${ }^{5}$ Bien que la méningite puisse survenir après une ponction de la dure-mère, elle est généralement secondaire à une infection systémique. Des abcès épiduraux se sont développés chez des parturientes après une épidurale continue. ${ }^{6}$ Un cas de méningite consécutif à un bloc CSE pour l'analgésie pendant le travail a aussi récemment été rapporté. ${ }^{7}$ Il n'est pas surprenant non plus d'apprendre que des cas d'infection sont survenus après l'épidurale continue prolongée administrée pour l'analgésie ainsi qu'après l'injection de stéroïdes. Par contre, de nombreux comptes rendus nous montrent que l'épidurale a été 
utilisée à long terme sans infections, et même d'autres qui soutiennent que l'anesthésie épidurale peut être administrée sans problèmes en présence d'une infection. ${ }^{9}$ Nous devons nous demander si ces cas ont quelque chose en commun et si nous pouvons faire quelque chose pour prévenir l'infection après l'anesthésie épidurale, sous-arachnoïdien et combinée.

En ce qui concerne les facteurs de risque de l'infection, ils sont soit extrinsèques soit intrinsèques. Les facteurs extrinsèques sont en rapport avec la technique de pénétration des espaces paraspinaux, le type de médicament et la durée du traitement. L'état général du patient, l'immunosuppression et la présence de maladies systémiques comme le diabète sont décrits comme facteurs intrinsèques. ${ }^{10}$ On peut constater, à la lecture des observations sur l'infection, qu'elles ont peu en commun. Certain(e)s des patient(e)s sont jeunes et en bonne santé (souvent des parturientes), d'autres sont malades et souffrent de maladies polysystémiques. Quand nous utilisons l'anesthésie épidurale et/ou sousarachnoïdienne, il faut avoir à l'esprit l'état du patient et une possibilité d'infection plus élevée chez le sujet affaibli, traité aux immunosuppresseurs ou diabétique. Ces états ne sont toutefois pas en eux-mêmes des contre-indications à la technique, et souvent, c'est même le contraire. Bromage a reconmandé l'usage du système medic-alert dans le cas des patients déjà traités aux stéroïdes épiduraux ${ }^{4}$ mais la faisabilité de cette entreprise était contestée autant sous son aspect pratique qu'économique dans l'éditorial qui accompagnait l'article. ${ }^{3} \mathrm{La}$ vigilance et l'examen physique peuvent très bien suffire. Les cas d'abcès épiduraux ayant mal évolués semblent d'abord avoir échappé au diagnostic et ensuite avoir été pris en charge trop tard. Les catastrophes peuvent être évitées même si l'infection elle-même ne peut l'être.

Il faut rechercher soigneusement les facteurs extrinsèques. Dans le cas présent, et dans d'autres, toutes les précautions aseptiques avaient été prises. Ceci incluait le brossage, le port des gants, de la blouse et du masque par l'exécutant, et le badigeonnage du patient avec des solutions aseptiques standards qu'on avait laissé sécher avant de faire la ponction. Malgré tout, il y a eu infection. Peut-on attribuer à la technique l'apparition d'un abcès épidural ou d'une méningite ? Une enquête récente auprès des anesthésistes obstétricaux britanniques a révélé que le masque n'était pas toujours porté. ${ }^{11}$ Nous ne possédons toutefois aucune donnée sur le port du masque et sa relation avec l'infection.

L'université McMaster vient tout juste de compléter une étude aléatoire sur le lavage des mains avec une brosse comparé au lavage des mains avec un savon antiseptique seul. Le brossage systématique des mains a été cessé dans nos salles d'opération pendant une année, sans que l'incidence des infections postopératoires ne change. Certains individus qui se lavaient les mains sans brossage avaient des décomptes bactériens inférieurs à ceux qui utilisaient une brosse. ${ }^{12} \mathrm{~J}$ 'ai aussi recherché s'il existait des études aléatoires sur l'utilisation de filtres avec les cathéters épiduraux. Ces filtres éliminent les particules comme le verre mais je n'ai pu corroborer qu'ils prévenaient la contamination bactérienne et secondairement la formation d'abcès dans l'espace épidural. Les solutions antiseptiques doivent être stériles. Les flacons d'iode à usages multiples sont parfois contaminés et les films de polyuréthane appliqués sur le site de la ponction augmentent la transpiration et favorisent la multiplication des bactéries. Nous utilisons des rubans adhésifs micropore alcoolisés pour recouvrir le site de l'épidurale. Ces rubans adhèrent très fortement à la peau tout en lui permettant de «respirer». L'influence de la durée d'une épidurale sur l'infection est indéterminée. Nous laissons le cathéter en place pour soulager la douleur postopératoire jusqu'à ce que la patiente puisse absorber des médicaments par la bouche, ce qui prend en moyenne quatre jours. Pendant neuf ans, nous n'avons pas constaté un seul abcès épidural. Quand il s'agit de cancéreux, nous laissons souvent un cathéter épidural en place pendant des semaines.

Il est tout à fait raisonnable d'utiliser une technique stérile quand on pénètre dans un des espaces du système nerveux central. Pour nous, ceci signifie l'utilisation d'un masque et de gants après lavage avec une solution antiseptique et assèchement avec une serviette stérile. L'uniforme habituel de travail en salle d'opération est porté mais pas nécessairement la blouse. Le dos du patient est badigeonné avec une solution antiseptique stérile et une fois sec, essuyé pour éviter de pousser le produit chimique dans l'espace épidural. Si le tissu cutané est perforé à plusieurs reprises et traumatisé, il serait sage de changer d'aiguille avant de pénétrer dans l'espace épidural ou sous-arachnoïdien. Je préfère installer un filtre antibactérien, surtout si le cathéter reste en place pendant des jours et que de multiples injections sont pratiquées. Nous changeons par la suite le filtre aux $48 \mathrm{~h}$. L'efficacité de ces filtres n'a pas été prouvée jusqu'à maintenant.

Avant tout, le patient doit être renseigné au sujet des risques et des avantages. Le consentement éclairé comprend la révélation des risques matériels. Malgré sa rareté, l'infection compliquée de séquelles neurologiques constitue un risque matériel. Quand les patientes le demandent, j'ai l'habitude de leur men- 
tionner que le risque d'infection ou d'hémorragie associé aux techniques épidurales et rachidiennes est toujours présent, mais éloigné et que les avantages dépassent largement ce risque. Au Canada, un consentement spécifique n'est pas requis avant l'administration de l'anesthésie pour une intervention. La visite préopératoire fournit l'opportunité de discuter des autres modes d'anesthésie disponibles et c'est le moment d'élaborer sur les techniques régionales. Aucun cas d'abcès épidural associé l'anesthésie régionale administrée pour l'anesthésie ou l'analgésie n'a jamais donné lieu eu à des poursuites au Canada.

L'observation actuelle nous confirme qu'après l'anesthésie épidurale ou sous-arachnoïdienne une infection est toujours possible. Nous devons donc prendre toutes les précautions raisonnables pour prévenir l'infection, en plus de connaitre les patients à risque, et d'exercer une surveillance très étroite. Les patients susceptibles devraient avoir été informés au sujet des signes de l'infection lorsqu'ils quittent le milieu hospitalier parce que les manifestations de l'abcès épidural surviennent quelquefois tardivement. Un diagnostic précoce et un traitement en urgence sont essentiels pour la prévention des séquelles désastreuses.

\section{References}

1 Schröter J, wa Djamba D, Hoffmann V, Bach A, Motsch $J$. Epidural abscess after combined spinal-epidural block. Can J Anaesth 1997; 44: 300-304.

2 Chestnut DH. Spinal anesthesia in the febrile patient (Editorial). Anesthesiology 1992; 76: 667-9.

3 Wildsmith JAW. Extradural abscess after central neural block (Editorial). Br J Anaesth 1993; 70: 387-8.

4 Carson D, Wildsmith JAW. The risk of extradural abscess. Br J Anaesth 1995; 75: 520-1.

5 Bromage PR. Spinal extradural abscess: pursuit of vigilance. Br J Anaesth 1993; 70: 471-3.

6 Ngan Kee WD, Jones MR, Thomas P, Worth RJ. Extradural abscess complicating extradural anaesthesia for Caesarean section. Br J Anaesth 1992; 69: 647-52.

7 Cascio $M$, Heath $G$. Meningitis following a conbined spinal-epidural technique in a labouring term parturient. Can J Anaesth 1996; 43: 399-402.

8 DuPen, SL, Peterson DG, Williams A, Bogosian AJ. Infection during chronic epidural catheterization: diagnosis and treatment. Anesthesiology 1990; 73: 905-9.

9 Jakobsen $K B$, Christensen, $M-K$, Carlsson PS. Extradural anaesthesia for repeated surgical treatment in the presence of infection. Br J Anaesth 1995; 75: 536-40.

10 Mahendru V, Bacon DR, Lema MJ. Multiple epidural abscesses and spinal anesthesia in a diabetic patient. Reg Anesth 1994; 19: 66-8.
11 Panikkar KK, Yentis SM. Wearing of masks for obstetric regional anaesthesia. A postal survey. Anaesthesia 1996; 51: 398-400.

12 Loeb MB, Wilcox L, Smaill F, Walter S, Boukhedouma Z. A randomized trial of surgical scrubbing with a brush compared to antiseptic soap alone. Am J Infect Control. (In press) 